\title{
The Price of Anarchy of the Proportional Allocation Mechanism Revisited
}

\author{
José R. Correa ${ }^{1}$, Andreas S. Schulz ${ }^{2}$, and Nicolás E. Stier-Moses ${ }^{3,4}$ \\ 1 Universidad de Chile, Santiago, Chile \\ correa@uchile.cl \\ 2 Massachusetts Institute of Technology, Cambridge, MA, USA \\ schulz@mit.edu \\ 3 Columbia University, New York, NY, USA \\ stier@gsb.columbia.edu \\ 4 Universidad Torcuato Di Tella, Buenos Aires, Argentina
}

\begin{abstract}
We consider the proportional allocation mechanism first studied by Kelly (1997) in the context of congestion control algorithms for communication networks. A single infinitely divisible resource is to be allocated efficiently to competing players whose individual utility functions are unknown to the resource manager. If players anticipate the effect of their bids on the price of the resource and their utility functions are concave, strictly increasing and continuously differentiable, Johari and Tsitsiklis (2004) proved that the price of anarchy is $4 / 3$. The question was raised whether there is a relationship between this result and that of Roughgarden and Tardos (2002), who had earlier shown exactly the same bound for nonatomic selfish routing with affine-linear congestion functions. We establish such a relationship and show, in particular, that the efficiency loss can be characterized by precisely the same geometric quantity. We also present a new variational inequality characterization of Nash equilibria in this setting, which enables us to extend the priceof-anarchy analysis to important classes of utility functions that are not necessarily concave.
\end{abstract}

\section{Introduction}

In a pioneering paper1], Roughgarden and Tardos [9] established that the loss of efficiency caused by selfish behavior in a multicommodity-flow network with affine-linear latency functions is at most 33\%, if compared to the cost of a systemoptimal solution. Shortly thereafter, in another widely cited paper, Johari and Tsitsiklis [5] observed virtually the same price of anarchy in a completely different context, in which a finite number of bidders with concave, strictly increasing and continuously differentiable utility functions compete for a single divisible resource, which is allocated in proportion to the bids, as suggested by Kelly [6].

1 http://www .acm.org/press-room/news-releases/2012/goedel-prize-2012

Y. Chen and N. Immorlica (Eds.): WINE 2013, LNCS 8289, pp. 109-120, 2013.

(C) Springer-Verlag Berlin Heidelberg 2013 
From the get-go, the question arose whether the sameness of the two bounds was just a coincidence 2 For instance, Johari and Tsitsiklis [5, Page 418] write:

However, it remains an open question whether a relationship can be drawn between the two games; in particular, we note that while [our main theorem] holds even if the utility functions are nonlinear, Roughgarden and Tardos have shown that the price of anarchy in traffic routing may be arbitrarily high if link latency functions are nonlinear.

In this paper, we offer an explanation as to why the upper bound on the price of anarchy is the same in both situations. We show that the Johari-Tsitsiklis bound follows from the same geometric quantity that describes the price of anarchy in the selfish-routing game that was analyzed by Roughgarden and Tardos and, for more general latency functions, by Roughgarden [8]. In earlier work [1, the authors of this paper had shown that the price of anarchy in the latter setting is bounded by $1 /(1-\beta(\mathcal{U}))$, where $\mathcal{U}$ is the class of (latency) functions considered and

$$
\beta(\mathcal{U})=\sup _{u \in \mathcal{U}} \sup _{0 \leq x \leq y \leq 1} \frac{x(u(y)-u(x))}{y u(y)},
$$

as long as the functions in $\mathcal{U}$ are nonnegative, nondecreasing and continuous 3 For a specific function $u$ and specific values $x \leq y$, it is easy to see that the numerator in the expression above is equal to the area of the shaded rectangle in Figure 1, while the denominator corresponds to the area of the big rectangle. In particular, it follows immediately from elementary geometric arguments that this ratio never exceeds $1 / 4$ for affine-linear functions, leading to a bound of $4 / 3$ on the price of anarchy. In fact, it was already noted in [1] that this remains true for more general classes of functions, including concave functions.

We show that the price of anarchy of the proportional allocation mechanism of Kelly [6] is bounded by $1 /(1-\beta(\mathcal{U}))$ as well. Here, we assume that all $u \in \mathcal{U}$ are concave, strictly increasing and continuously differentiable, as did Johari and Tsitsiklis [5], In particular, we get the same bound of $4 / 3$. In addition, our proof is considerably simpler than Johari and Tsitsiklis' original proof, and it follows

${ }^{2}$ Koutsoupias and Papadimitriou [7] introduced the price of anarchy as the worstcase ratio of the cost of an equilibrium to that of an optimum. In particular, in the minimization context of Roughgarden and Tardos, the price of anarchy is $4 / 3$. Johari and Tsitsiklis considered a maximization problem and established a worstcase efficiency loss of $25 \%$. For reasons of consistency, we assume that the price of anarchy in a maximization setting is defined as the worst-case ratio of the value of an optimum to that of an equilibrium. In particular, for us, the price of anarchy is always greater than or equal to one.

3 As noted in [1], $1 /(1-\beta(\mathcal{U}))$ is equal to the price-of-anarchy value $\alpha(\mathcal{U})$ presented in 8 , but the use of $\beta(\mathcal{U})$ allows for the inclusion of more general functions and the geometric interpretation first pointed out in [2].

4 This assumption ensures the existence and uniqueness of a Nash equilibrium, as long as there are at least two players; see [4] for details. 


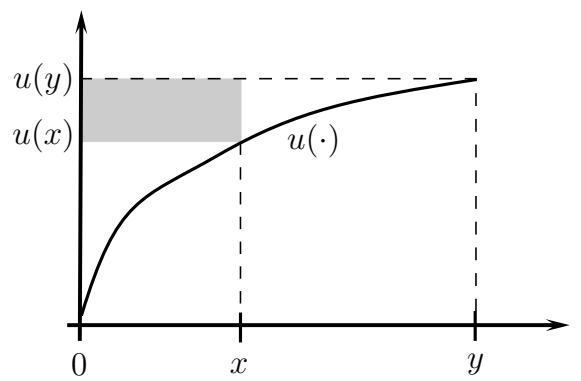

Fig. 1. The geometric interpretation of $\beta(\mathcal{U})$ as the supremum of the shaded area over the area of the big rectangle defined by the origin and the point $(y, u(y))$

the same steps as our earlier proof for the price of anarchy in selfish routing: The key inequality is delivered by a variational inequality derived from the optimality conditions of a concave program. Roughgarden [4. Proof of Theorem 21.4] used the same idea for the proportional allocation mechanism, but relied on a different concave program, which does not lend itself to the same quantity $1 /(1-\beta(\mathcal{U}))$ that arises in the context of selfish routing. Moreover, both the original proof by Johari and Tsitsiklis and Roughgarden's proof make explicit use of the concavity of the utility functions, whereas ours does not, allowing us to extend the analysis of the proportional allocation mechanism to situations in which utility functions are not necessarily concave. For this, we present a characterization of equilibria by a new variational inequality, which holds true as long as the second derivative of the utility functions does not become too positive. This condition encompasses certain convex functions, allowing us to capture some aspects of economies of scale. Corresponding functions include specific polynomials, exponential functions and queueing delay functions, which all give rise to a constant price of anarchy.

\section{The Proportional Allocation Mechanism}

Johari and Tsitsiklis [5] consider the following model. There is a single divisible resource shared by a set $N$ of players 5 Each player $i \in N$ has a concave, strictly increasing and continuously differentiable utility function $U_{i}:[0,1] \rightarrow \mathbb{R}_{+}$, so that her utility from receiving a fraction $x_{i}$ of the resource equals $U_{i}\left(x_{i}\right) 6$ In a utilitarian setting, the resource would ideally be allocated so as to maximize the total utility:

$$
\max _{x \in \Delta} \sum_{i \in N} U_{i}\left(x_{i}\right),
$$

\footnotetext{
${ }^{5}$ To exclude pathological cases, we assume throughout the paper that $|N| \geq 2$.

${ }^{6}$ For convenience, it is assumed that utility is measured in monetary units.
} 
where $\Delta:=\left\{x \in \mathbb{R}_{+}^{N}: \sum_{i \in N} x_{i} \leq 1\right\}$. However, in order to implement this solution, the resource manager would need to have knowledge of all utility functions, which is often not realistic. Alternatively, one could use Kelly's proportional allocation mechanism [6], where each player $i \in N$ bids a nonnegative amount $v_{i}$, which is going to be her payment, and obtains a fraction of the resource equal to $x_{i}=v_{i} / \sum_{j \in N} v_{j}$. In particular, player $i$ 's payoff is equal to

$$
U_{i}\left(x_{i}\right)-v_{i}=U_{i}\left(\frac{v_{i}}{\sum_{j \in N} v_{j}}\right)-v_{i}
$$

and we assume that her payoff is zero if all players bid zero. Player $i$ wants to maximize this expression, and an equilibrium is a vector of bids such that each player bids optimally, given the bids of the other players. Hajek and Gopalakrishnan [3] proved that there exists a unique equilibrium. Moreover, it is not hard to see (e.g., [5. Proof of Theorem 2]) that a vector $v \in \mathbb{R}^{N}$ is an equilibrium if and only if, for all $i \in N$,

$$
\left(1-x_{i}^{\mathrm{NE}}\right) U_{i}^{\prime}\left(x_{i}^{\mathrm{NE}}\right)=V \text { if } v_{i}>0 \text {, and } U_{i}^{\prime}(0) \leq V \text { if } v_{i}=0
$$

Here, $V=\sum_{j \in N} v_{j}$ and $x_{i}^{\mathrm{NE}}=v_{i} / V$.

\section{A New Proof for the Price of Anarchy}

We now introduce a new concave program and show that the equilibrium allocation $x^{\mathrm{NE}}$ is an optimal solution. Consider

$$
\max _{x \in \Delta} \sum_{i \in N}\left(1-x_{i}^{\mathrm{NE}}\right) U_{i}\left(x_{i}\right)
$$

where $x_{i}^{\mathrm{NE}}$ is fixed in the objective function. The optimality conditions are:

$$
\begin{aligned}
\left(1-x_{i}^{\mathrm{NE}}\right) U_{i}^{\prime}\left(x_{i}\right) & =\lambda+\mu_{i} & & \text { for all } i \in N, \\
\sum_{i \in N} x_{i} & \leq 1, & & \\
\mu_{i} x_{i} & =0 & & \text { for all } i \in N, \\
\mu_{i} & \leq 0 & & \text { for all } i \in N, \\
x_{i} & \geq 0 & & \text { for all } i \in N, \\
\lambda & \geq 0 . & &
\end{aligned}
$$

By taking $\lambda=V$ and $\mu_{i}=U_{i}^{\prime}(0)-V$ when $x_{i}^{\mathrm{NE}}=0$, it follows that $x^{\mathrm{NE}}$ satisfies the Karush-Kuhn-Tucker conditions and, thus, is a maximum of (2).

Using the optimality of $x^{\mathrm{NE}}$ for (2), the monotonicity of the utility functions and the definition of $\beta(\mathcal{U})$, we are ready to prove the desired bound on the price of anarchy of the proportional allocation mechanism by Kelly. Here, $x^{*} \in \Delta$ is 
an arbitrary feasible vector, and the result follows when $x^{*}$ is taken to be the socially optimal assignment:

$$
\begin{aligned}
\sum_{i \in N} U_{i}\left(x_{i}^{\mathrm{NE}}\right) & \geq \sum_{i \in N}\left(x_{i}^{\mathrm{NE}} U_{i}\left(x_{i}^{\mathrm{NE}}\right)+\left(1-x_{i}^{\mathrm{NE}}\right) U_{i}\left(x_{i}^{*}\right)\right) \\
& \geq \sum_{i \in N} U_{i}\left(x_{i}^{*}\right)-\sum_{i \in N: x_{i}^{*}>x_{i}^{\mathrm{NE}}} x_{i}^{\mathrm{NE}}\left(U_{i}\left(x_{i}^{*}\right)-U_{i}\left(x_{i}^{\mathrm{NE}}\right)\right) \\
& \geq \sum_{i \in N} U_{i}\left(x_{i}^{*}\right)-\sum_{i \in N: x_{i}^{*}>x_{i}^{\mathrm{NE}}} \beta(\mathcal{U}) x_{i}^{*} U_{i}\left(x_{i}^{*}\right) \\
& \geq(1-\beta(\mathcal{U})) \sum_{i \in N} U_{i}\left(x_{i}^{*}\right) .
\end{aligned}
$$

We have given a new proof of the upper bound in the following theorem and, at the same time, established a connection to the quantity $\beta(\mathcal{U})$, which plays a similar role in the price of anarchy of selfish routing, as discussed in the introduction.

Theorem 1 (Johari and Tsitsiklis 2004). The price of anarchy for the proportional allocation mechanism is $4 / 3$ when utility functions are strictly increasing, continuously differentiable and concave.

\section{A Variational Inequality for the Nonconcave Case}

In the derivation of the price of anarchy for the proportional allocation mechanism in Section 3 we made use of a new variational inequality obtained easily from the optimality conditions of the concave program (2):

$$
\sum_{i \in N}\left(1-x_{i}^{\mathrm{NE}}\right)\left(U_{i}\left(x_{i}^{\mathrm{NE}}\right)-U_{i}\left(x_{i}\right)\right) \geq 0 \text { for all } x \in \Delta .
$$

We will now derive another variational inequality that continues to characterize equilibria even if the players' utility functions are not concave anymore. This allows us to extend the price-of-anarchy analysis to settings that include economies of scale and other situations in which players' utilities may not be concave.

Johari and Tsitsiklis [5] characterized equilibria as optimal solutions to the following nonlinear program:

$$
\max _{x \in \Delta} \sum_{i \in N}\left(\left(1-x_{i}\right) U_{i}\left(x_{i}\right)+\int_{0}^{x_{i}} U_{i}(z) d z\right) .
$$

The partial derivative of the objective function in direction $x_{i}$ is $\left(1-x_{i}\right) U_{i}^{\prime}\left(x_{i}\right)$, giving exactly the equilibrium conditions shown in (11). The first-order optimality conditions of Problem (4) can be written as a variational inequality: An equilibrium allocation $x^{\mathrm{NE}}$ is characterized by

$$
\sum_{i \in N}\left(1-x_{i}^{\mathrm{NE}}\right) U_{i}^{\prime}\left(x_{i}^{\mathrm{NE}}\right)\left(x_{i}^{\mathrm{NE}}-x_{i}\right) \geq 0 \quad \text { for all } x \in \Delta .
$$


This is exactly the variational inequality used by Roughgarden 4, Proof of Theorem 21.4] in his proof of the price-of-anarchy result of Johari and Tsitsiklis [5]. In their setting, concavity and monotonicity of the utility functions $U_{i}$ guarantee that the objective function in (4) is concave, making sure that the optimality conditions of the nonlinear program characterize globally optimal solutions. We will exploit the fact that concavity of this objective can still be guaranteed by less stringent assumptions on $U_{i}$. For convenience, we now assume that each $U_{i}$ is twice-differentiable (and we continue to assume that it is strictly increasing). Then, for the objective function of (4) to be concave over the feasible region $\Delta$, it suffices if, for all $i \in N$,

$$
\frac{U_{i}^{\prime \prime}(x)}{U_{i}^{\prime}(x)} \leq \frac{1}{1-x} \quad \text { for } 0 \leq x<1
$$

If this is the case, an equilibrium continues to exist, it is unique, and it is still characterized by the first-order optimality conditions of (4). Notice that (6) is indeed a relaxation of concavity, because for strictly increasing, concave functions, the left-hand side is not positive. In fact, Condition (6) is satisfied by certain convex functions, such as some polynomials, or exponential functions, or wait functions of queueing networks, such as $(c-x)^{-1}$ for $c \geq 2$.

Assuming (6), the following nonlinear program is concave and has exactly the same first-order optimality conditions as (4), as one can easily check by taking the derivative of the objective function:

$$
\max _{x \in \Delta} \sum_{i \in N}\left(U_{i}\left(x_{i}\right)-\int_{U_{i}(0)}^{U_{i}\left(x_{i}\right)} U_{i}^{-1}(z) d z\right) .
$$

Here, $U_{i}^{-1}$ is the inverse function of $U_{i}$. In turn, after a change of variables, $y_{i}=U_{i}\left(x_{i}\right)$, this problem is equivalent to

$$
\max _{y: y_{i} \geq U_{i}(0), \sum_{i} U_{i}^{-1}\left(y_{i}\right) \leq 1} \sum_{i \in N}\left(y_{i}-\int_{0}^{y_{i}} U_{i}^{-1}(z) d z\right) .
$$

The optimal solution $y^{\mathrm{NE}}$ of (77) is equal to the vector $\left(U_{i}\left(x_{i}^{\mathrm{NE}}\right)\right)_{i \in N}$, because $x^{\mathrm{NE}}$ is the unique solution to (4), and all the nonlinear programs above are equivalent. If all utility functions are concave, $y^{\mathrm{NE}}$ satisfies the first-order optimality conditions

$$
\sum_{i \in N}\left(U_{i}^{-1}\left(y_{i}^{\mathrm{NE}}\right)-1\right)\left(y_{i}-y_{i}^{\mathrm{NE}}\right) \geq 0
$$

for all vectors $y$ that are feasible for the constraints of (7). Undoing the change of variables, this variational inequality is equivalent to (3), and we have provided an alternative way of deriving variational inequality (3). In case of nonconcave utility functions that satisfy (6), we can use the following variational inequality to characterize equilibria, which follows directly from (7):

$$
\sum_{i \in N}\left(y_{i}^{\mathrm{NE}}-y_{i}\right)-\left(\sum_{i \in N} \int_{y_{i}}^{y_{i}^{\mathrm{NE}}} U_{i}^{-1}(z) d z\right) \geq 0
$$


for all vectors $y$ that are feasible for the constraints of (7). We will use this variational inequality in the next section to derive price-of-anarchy bounds for non-concave utility functions satisfying (6).

\section{The Price of Anarchy in the Nonconcave Case}

From now on, we will work with the relaxed concavity assumption (6), which suffices for the variational inequality (8) to hold. We will compute the price of anarchy in this more general setting. For this, we introduce a new constant "beta" that depends again just on the class of utility functions considered:

$$
\hat{\beta}(\mathcal{U}):=\sup _{u \in \mathcal{U}} \sup _{u(0) \leq y \leq u(1)} \frac{\int_{u(0)}^{y} u^{-1}(z) d z}{y} .
$$

With this definition and variational inequality (8) in place, we bound the price of anarchy of the proportional allocation mechanism using an approach similar to that in Section 3. only that we have one less inequality in the derivation:7

$$
\begin{aligned}
\sum_{i \in N} U_{i}\left(x_{i}^{\mathrm{NE}}\right) & \geq \sum_{i \in N} U_{i}\left(x_{i}^{*}\right)-\sum_{i \in N: x_{i}^{*}>x_{i}^{\mathrm{NE}}} \int_{U_{i}\left(x_{i}^{\mathrm{NE}}\right)}^{U_{i}\left(x_{i}^{*}\right)} U_{i}^{-1}(z) d z \\
& \geq(1-\hat{\beta}(\mathcal{U})) \sum_{i \in N} U_{i}\left(x_{i}^{*}\right) .
\end{aligned}
$$

Theorem 2. The price of anarchy for the proportional allocation mechanism is at most $1 /(1-\hat{\beta}(\mathcal{U}))$, when all utility functions belong to a family $U$ of strictly increasing and twice differentiable functions that satisfy (6).

It remains to compute the actual value of $\hat{\beta}$ for concrete families $\mathcal{U}$ of this kind, which is the content of the next section.

\section{Computing $\hat{\boldsymbol{\beta}}$}

For the calculation of $\hat{\beta}$, we will parameterize the family of nonnegative, strictly increasing, twice differentiable utility functions that satisfy (6) as follows: For $c \geq 1$, we let $\mathcal{U}^{c}$ be the set of all such functions $U_{i}$ for which

$$
\left(1-\frac{x}{c}\right) U_{i}^{\prime \prime}(x) \leq U_{i}^{\prime}(x) \text { for } 0 \leq x \leq 1 .
$$

\footnotetext{
7 This happens because, compared to the definition of $\beta$, the product in the denominator is replaced by a single value. Interestingly, had we defined $\hat{\beta}$ with $y u^{-1}(y)$ in the denominator, its geometric interpretation would have been that we are seeking an upper bound on the ratio of the area defined by the integral in the numerator to the area of the rectangle defined by the origin and the point $\left(u^{-1}(y), y\right)$, which is easily seen to be at most $1 / 2$ for concave functions (see Figure 21). While this would suffice to replicate the "easy [...] bound" of Johari and Tsistsiklis [5, Page 415], $\hat{\beta}$ as defined here has the potential to lead to stronger results.
} 


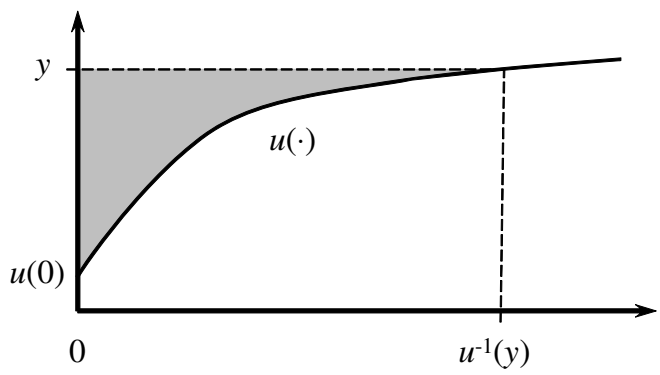

Fig. 2. The geometric meaning of the numerator in the definition of $\hat{\beta}(\mathcal{U})$

It is straightforward to see that $\mathcal{U}^{1}$ is equal to the set of all functions that satisfy (6), while $\mathcal{U}^{\infty}$ contains the functions that satisfy

$$
U_{i}^{\prime \prime}(x) \leq U_{i}^{\prime}(x) \text { for } 0 \leq x \leq 1
$$

We begin with the computation of $\hat{\beta}\left(\mathcal{U}^{\infty}\right)$, which amounts to computing

$$
\sup _{u: u(0) \geq 0, u^{\prime} \geq 0, u^{\prime \prime} \leq u^{\prime}} \sup _{u(0) \leq y \leq u(1)} \frac{\int_{u(0)}^{y} u^{-1}(z) d z}{y} .
$$

Note that for any fixed function $u$ the supremum over $y$ is attained at $y=u(1)$ since the derivative of the argument equals

$$
\frac{u^{-1}(y) y-\int_{u(0)}^{y} u^{-1}(z) d z}{y^{2}},
$$

which is greater than zero as $u^{-1}$ is strictly increasing. Therefore we have that

$$
\hat{\beta}\left(\mathcal{U}^{\infty}\right)=\sup _{u: u(0) \geq 0, u^{\prime} \geq 0, u^{\prime \prime} \leq u^{\prime}} \frac{\int_{u(0)}^{u(1)} u^{-1}(z) d z}{u(1)} .
$$

Note that we may assume $u(0)=0$; otherwise subtracting $u(0)$ from a given function will maintain feasibility and only increase the supremum. Also, we may assume $u(1)=1$ since, when dividing a function $u$ by this quantity, the areas that represent the denominator and the numerator will shrink by the same factor, and, of course, feasibility will not be affected. We conclude that:

$$
\hat{\beta}\left(\mathcal{U}^{\infty}\right)=\sup _{u: u(0)=0, u(1)=1, u^{\prime} \geq 0, u^{\prime \prime} \leq u^{\prime}} \int_{0}^{1} u^{-1}(z) d z .
$$

Therefore, solving the differential equation $u^{\prime \prime}(z)=u^{\prime}(z)$ with the initial values $u(0)=0$ and $u(1)=1$ provides a feasible solution to our problem, given by $u(z)=\left(e^{z}-1\right) /(e-1)$. We now prove that this is optimal. 
To maximize the area in the supremum we need to find a function $u$ with the required properties that is as small as possible for any given $0<z<1$. However, in principle this function may not exist, as for different values of $z$ we may have different functions being the smallest, so we define the function $g:[0,1] \rightarrow \mathbb{R}_{+}$ by

$$
g(z):=\inf _{u: u(0)=0, u(1)=1, u^{\prime} \geq 0, u^{\prime \prime} \leq u^{\prime}} u(z) .
$$

Clearly $\hat{\beta}\left(\mathcal{U}^{\infty}\right) \leq \int_{0}^{1} g^{-1}(z) d z$, so it remains to compute $g$ to provide a matching upper bound. Note that we may interpret $u(\cdot)$ as a cumulative distribution function (cdf). Calling its pdf $h$, we have that

$$
g(z)=\inf _{h \text { density s.t. } h^{\prime} \leq h} \int_{0}^{z} h(t) d t .
$$

We complete the argument by proving that $g(z)=\left(e^{z}-1\right) /(e-1)$. To get a contradiction, suppose that $g(z)<\left(e^{z}-1\right) /(e-1)$ and let us consider $f(z):=$ $e^{z} /(e-1)$. Then, there exists a density $h$, such that $h^{\prime} \leq h$, satisfying that

$$
\int_{0}^{z} h(t) d t<\frac{e^{z}-1}{e-1}=f(z)-f(0)=\int_{0}^{z} f(t) d t .
$$

Thus, there exists a point $z_{1}<z$ for which $h\left(z_{1}\right)<f\left(z_{1}\right)$, and, as both $h$ and $f$ are density functions, $\int_{0}^{1} h(t) d t=\int_{0}^{1} f(t) d t$, so that we may consider $z_{2}$ as the smallest point larger than $z_{1}$ for which $h\left(z_{2}\right)=f\left(z_{2}\right)$. Thus $h$ is smaller than $f$ in the interval $\left[z_{1}, z_{2}\right)$, and they are equal at $z_{2}$. Since in this interval $h$ grows more than $f$, it is immediate that there exists a point $x \in\left[z_{1}, z_{2}\right)$ for which $h^{\prime}(x)>f^{\prime}(x)$. But on the other hand $f(x)=f^{\prime}(x)$ and $h(x) \geq h^{\prime}(x)$, implying that $h(x)>f(x)$. A contradiction follows.

We conclude that $g(z)=\left(e^{z}-1\right) /(e-1)$ and thus

$$
\hat{\beta}\left(\mathcal{U}^{\infty}\right)=1-\int_{0}^{1} \frac{e^{z}-1}{e-1} d z=\frac{1}{e-1} \approx 0.581977,
$$

yielding an upper bound of 2.392213 on the price of anarchy.

We now use a similar approach to compute $\hat{\beta}\left(\mathcal{U}^{c}\right)$. Note first that the solution to the differential equation $(1-z / c) u^{\prime \prime}(z)=u^{\prime}(z)$ with initial values $u(0)=0$ and $u(1)=1$, for an arbitrary $c>1$, is given by

$$
\bar{u}(z)=\frac{(c-z)^{1-c}-c^{1-c}}{(c-1)^{1-c}-c^{1-c}} .
$$

Therefore $\hat{\beta}\left(\mathcal{U}^{c}\right) \geq \int_{0}^{1} \bar{u}^{-1}(z) d z$. We now prove that this is actually an equality. Again we consider the function $g:[0,1] \rightarrow \mathbb{R}_{+}$defined by

$$
g(z):=\inf _{u: u(0)=0, u(1)=1, u^{\prime} \geq 0,(1-z / c) u^{\prime \prime} \leq u^{\prime}} u(z),
$$

which allows us to interpret $u$ as a cdf. Calling its pdf $h$, we have that

$$
g(z)=\inf _{h \text { density s.t. }(1-z / c) h^{\prime} \leq h} \int_{0}^{z} h(t) d t .
$$


To get a contradiction, suppose that $g(z)<\bar{u}(z)$ and let us consider the density function

$$
f(z):=\bar{u}^{\prime}(z)=\frac{(c-1)(c-z)^{-c}}{(c-1)^{1-c}-c^{1-c}} .
$$

Then, there exists a density $h$, such that $(1-z / c) h^{\prime} \leq h$, satisfying that

$$
\int_{0}^{z} h(t) d t<\bar{u}(z)=\int_{0}^{z} f(t) d t .
$$

Thus, there exists a point $z_{1}<z$ for which $h\left(z_{1}\right)<f\left(z_{1}\right)$, and, as both $h$ and $f$ are density functions, $\int_{0}^{1} h(t) d t=\int_{0}^{1} f(t) d t$. We may therefore consider $z_{2}$ as the smallest point larger than $z_{1}$ for which $h\left(z_{2}\right)=f\left(z_{2}\right)$. Thus, $h$ is smaller than $f$ in the interval $\left[z_{1}, z_{2}\right)$, and they are equal at $z_{2}$. Since in this interval $h$ grows more than $f$, it is immediate that there exists a point $x \in\left[z_{1}, z_{2}\right)$ for which $h^{\prime}(x)>f^{\prime}(x)$. On the other hand, $f(x)=f^{\prime}(x)(1-x / c)$ and $h(x) \geq$ $h^{\prime}(x)(1-x / c)$, implying that $h(x)>f(x)$. A contradiction follows.

We conclude that $g(z)=\bar{u}(z)$ and thus

$$
\hat{\beta}\left(\mathcal{U}^{c}\right)=1-\int_{0}^{1} \bar{u}(z) d z,
$$

leading to an upper bound on the price of anarchy of

$$
\left(1-c+\frac{c-1}{c-2} \cdot \frac{(c-1)^{2-c}-c^{2-c}}{(c-1)^{1-c}-c^{1-c}}\right)^{-1} .
$$

The concrete numerical value as a function of $c$ can be seen in Table 1 and Figure 3 .

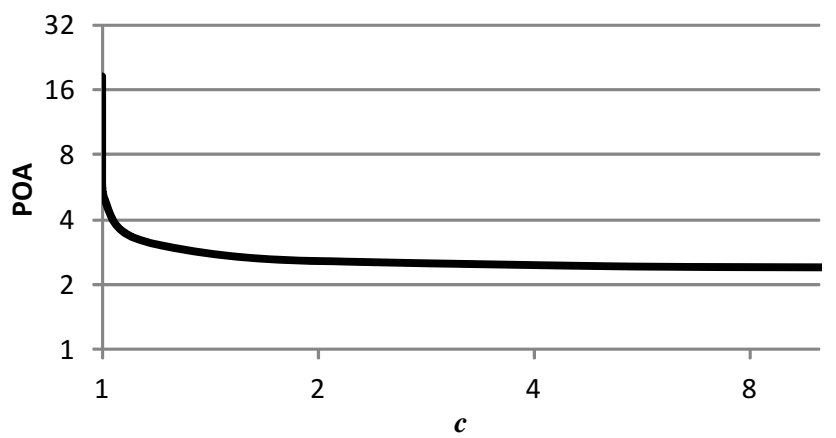

Fig. 3. The new bound on the price of anarchy as a function of $c \geq 1$ (log-log scale) 
Table 1. The new bound on the price of anarchy as a function of $c \geq 1$.

\begin{tabular}{rc}
$c$ & POA \\
\hline 1 & $\infty$ \\
1.000000001 & 20.72326624 \\
1.00000001 & 18.42068566 \\
1.0000001 & 16.11813311 \\
1.000001 & 13.81578405 \\
1.00001 & 11.51480886 \\
1.0001 & 9.222259832 \\
1.001 & 6.974091719 \\
1.01 & 4.907853238 \\
1.1 & 3.345367672 \\
1.5 & 2.732050808 \\
2 & 2.587079623 \\
5 & 2.449115044 \\
10 & 2.418357995 \\
20 & 2.404781333 \\
50 & 2.397126363 \\
100 & 2.394650571
\end{tabular}

\section{Concluding Remarks}

We have added a link between the price-of-anarchy analysis of the nonatomic selfish routing game with concave latency functions and that of the proportional allocation mechanism with concave utility functions. In both cases, the price of anarchy is governed by the same geometric quantity. We have also presented two new variational inequalities characterizing equilibria in the proportional allocation mechanism; one for the case of concave utility functions, and one that works for certain classes of nonconcave utility functions. This allowed us to give the first price-of-anarchy analysis of the proportional allocation mechanism with nonconcave utility functions. Even though we worked under the assumption that, for any $x \in(0,1)$, the ratio between the second and the first derivative of each utility function is at most $1 /(1-x)$, a closer look at this game reveals that it would actually suffice if that ratio were at most $2 /(1-x)$, which is something to be exploited in the future. We also leave as an open problem to determine tight bounds for the price of anarchy for the nonconcave classes of utility functions considered here. Finally, it is also worth mentioning that the concave problem (7) does not require the differentiability of the $U_{i}$ 's. One can actually prove that the optimal solutions to this problem always coincide with equilibria (a standard application of subdifferentials). Then variational inequality (3), which can be derived from (7), leads to the same price of anarchy in the concave, but not necessarily differentiable case.

Acknowledgements. This work was partially supported by Nucleo Milenio Información y Coordinación en Redes ICM/FIC P10-024F and by CONICET Argentina through grant PICT-2012-1324. 


\section{References}

1. Correa, J.R., Schulz, A.S., Stier-Moses, N.E.: Selfish routing in capacitated networks. Mathematics of Operations Research 29, 961-976 (2004)

2. Correa, J.R., Schulz, A.S., Stier-Moses, N.E.: A geometric approach to the price of anarchy in nonatomic congestion games. Games and Economic Behavior 64, 457-469 (2008)

3. Hajek, B., Gopalakrishnan, G.: Do greedy autonomous systems make for a sensible Internet? Presented at the Conference on Stochastic Networks, Stanford University, CA (2002)

4. Johari, R.: The price of anarchy and the design of scalable resource allocation mechanisms. In: Nisan, N., Roughgarden, T., Tardos, É., Vazirani, V.V. (eds.) Algorithmic Game Theory. Cambridge University Press (2007)

5. Johari, R., Tsitsiklis, J.N.: Efficiency loss in a network resource allocation game. Mathematics of Operations Research 29, 407-435 (2004)

6. Kelly, F.: Charging and rate control for elastic traffic. European Transactions on Telecommunications 8, 33-37 (1997)

7. Koutsoupias, E., Papadimitriou, C.: Worst-case equilibria. In: Meinel, C., Tison, S. (eds.) STACS 1999. LNCS, vol. 1563, pp. 404-413. Springer, Heidelberg (1999)

8. Roughgarden, T.: The price of anarchy is independent of the network topology. Journal of Computer and System Sciences 67, 341-364 (2003)

9. Roughgarden, T., Tardos, É.: How bad is selfish routing? Journal of the ACM 49, 236-259 (2002) 